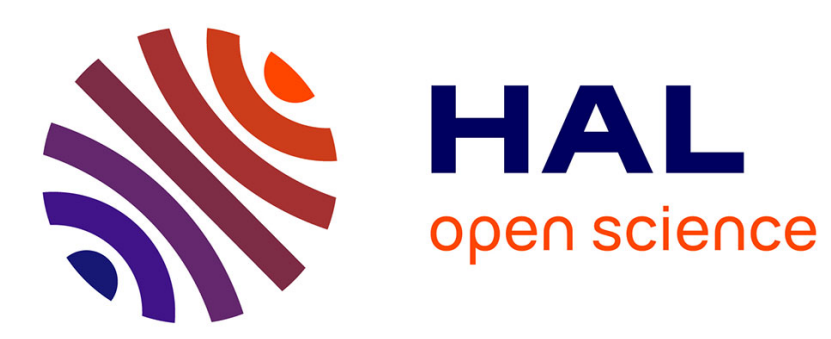

\title{
Simulation and control of three-dimensional wakes
}

Georges-Henri Cottet, Philippe Poncet

\section{To cite this version:}

Georges-Henri Cottet, Philippe Poncet. Simulation and control of three-dimensional wakes. Computers and Fluids, 2004, 33 (5-6), pp.697-713. 10.1016/j.compfluid.2003.01.001 . hal-02010683

\section{HAL Id: hal-02010683 \\ https://hal.science/hal-02010683}

Submitted on 4 Mar 2019

HAL is a multi-disciplinary open access archive for the deposit and dissemination of scientific research documents, whether they are published or not. The documents may come from teaching and research institutions in France or abroad, or from public or private research centers.
L'archive ouverte pluridisciplinaire HAL, est destinée au dépôt et à la diffusion de documents scientifiques de niveau recherche, publiés ou non, émanant des établissements d'enseignement et de recherche français ou étrangers, des laboratoires publics ou privés. 


\title{
Simulation and Control of Three-Dimensional Wakes
}

\author{
Georges-Henri Cottet ${ }^{\star}$, Philippe Poncet ${ }^{\dagger}$ \\ * LMC-IMAG Université Joseph Fourier, BP 53, 38041 Grenoble Cedex 9, France. \\ ${ }^{\dagger}$ Laboratoire MIP, Dept. GMM, INSA Toulouse, 135 avenue de Rangueil, 31077 Toulouse Cedex 4, France.
}

\begin{abstract}
We illustrate the problem of wake optimization on two examples. In the first example, the destruction of trailing vortices, we show that stochastic optimization is a flexible tool to identify vortex systems enhancing natural instabilities. In the second example, the problem of drag reduction in a cylinder wake, a priori information on the flow dynamics is highly desirable and we give some preliminary results which should be useful to select the most efficient parameters in an optimization strategy based on differential rotation of the cylinder.
\end{abstract}

Keywords : Three-dimensional flows; Wakes; Control; Transition to turbulence.

\section{Introduction}

The control of wakes is a subject of paramount importance in aircraft and automobile industry. Depending on the particular application, wake control can have various goals and can be achieved either by passive or active strategies. Passive control mostly operates through shape optimization and often results in the addition of appendices like foilers or ribblets to the surface of the obstacle. Active control implies that one is ready to impart energy on the flow by means of actuators on the surface of the obstacle, keeping in mind that this energy must be included in the global energy budget to conclude on the efficiency of the particular control strategy.

We focus here on two examples, each of them emphasizing a specific challenge of active control in 3D wakes. The first example is the destruction of trailing vortices shed by airplane wings. The second example is the drag reduction behind a bluff body. Both examples illustrate the need of active control. In the first case, there is little hope that any reasonable passive device could prevent the formation, or significantly modify the characteristics, of tip vortices that are created by the wings and flaps of an aircraft. In the second case, the automobile industry is a good example where shape optimization has led to important improvements in the last decades, but nowadays shows its limits, mostly due to design considerations. In the coming years, new drastic regulations in pollutants emissions will impose to explore new directions and in particular active control strategies.

These strategies, beside the technology issues that they will raise, will be very demanding in terms of simulation and optimization tools. Three-dimensional wakes are still a very challenging field for simulation methods, because of the complex unsteady features of the flows. Concerning optimization itself, if optimal control has been able to give interesting results for 2D flows $[9,17]$, there is still a long way to a systematic approach of 3D flows. Close loop control, where actuation would be imposed adaptively in terms of sensor informations is an even more open field, with the noticeable exception of [10] where it is shown that pressure measurements on the surface of the obstacle can be turned into vorticity fluxes and, ultimately, blowing and suction to significantly reduce the drag. Most of the current works in the field still aim at gaining insights into the dynamics of the flows in order to propose simple open loop strategies.

In this paper we review some recent or on-going efforts to implement evolution strategies for the optimization of 3D wakes. Evolution strategies are stochastic optimization methods where parameters are optimized through random search followed by selection on the basis of the evaluation of an objective function. Their 
convergence properties are not as well established as for gradient based methods, but they do not require to differentiate objective functions and are thus rather straightforward to implement. Other advantages are their ability to track global, instead of local, optima, their natural parallelism and their flexibility in accounting for constraints or a priori informations in the parameter space. The trade-off is clearly that they are CPU time consuming, in particular for the optimization of 3D unsteady flows, because their convergence is slow and every iteration involves a complete run. Evolution strategies have already proved to be very efficient for several instances of flow optimization. We refer to [19] and the references therein for examples in mixing and jet optimization.

In the sequel, we first show in section 2 that the destruction of trailing vortices is a striking case where evolution strategy is successful. The starting point here was a study by Crouch [5] of cooperative instabilities of two pairs of vortex tubes. In that study some particular parameter values where shown to potentially enhance vortex break-up, on a time scale much smaller than classical single-pair Crow instabilities. In this example, the problem setting is rather simple and simulations are not very expensive. Evolution strategies not only could rapidly converge to a solution very close to the particular parameters exhibited in [5], but also could open up to other, more efficient, solutions.

In section 3, we turn to the problem of drag reduction in a cylinder wake. In that case, in order to derive successful evolution strategies the complexity of the dynamics makes it crucial to reduce the parameter space dimension. One way to achieve this dimension reduction is to inject a priori informations available for this type of flows. Section 3 is devoted to that particular problem for the cylinder wake. We in particular investigate the links between three-dimensional features in the wake and drag values. We first consider two-dimensional control strategies, based on cylinder rotations then on 2D differential rotation parameters identified in [11]. We show that these control strategies on 3D flows make the flow return to a $2 \mathrm{D}$ state. Based on the 3D instability modes that naturally develop in uncontrolled 3D wakes, we then explore some 3D control profiles and discuss their potential efficiency in the context of drag optimization.

\section{Optimization of trailing vortices}

The control of trailing vortices remains a challenging problem of strong economical interest. These vortices are naturally shed by airplanes and cause strong down-wash that are a hazard for following aircrafts. Many studies have focused on the so-called Crow instability that spontaneously break-up these vortices [20]. Recently some studies $[5,18]$ have considered cooperative instabilities resulting from the interaction of several vortex pairs. The starting point of the study in [5] is the identification of a complex system of vortices shed by the wings, the flaps and the fuselage of a typical aircraft. This vortex system is sketched in Figure 1. The two pairs originating from the wing tips and the inboard flaps are of particular interest. Crouch [5] shows that, depending on the perturbations that are imparted to these two pairs of initially parallel tubes, several instability regimes can be observed, respectively termed long wave, transient growth, and short wave instabilities (Figure 1, right picture). In particular, the transient growth regime was shown to produce a growth rate far exceeding that of a single pair. Based on these findings Crouch and Spalart proposed to implement active control devices to trigger the proper instabilities. Our goal in this study was to identify the fastest growing instability by performing a systematic search in the parameter space defining the initial state of the perturbed vortex pair, having in mind that the success of such an approach could open up to the investigation of a broader class of vortex systems with the possibility of further accelerating the break-up process.

\subsection{The evolution strategy}

The principle of Evolution Strategies (ES) is an exploration of the parameter space by a random walk followed by a simple selection process. If $f$ is the function to minimize and $X \in R^{N}$ denotes the parameter vector, an 


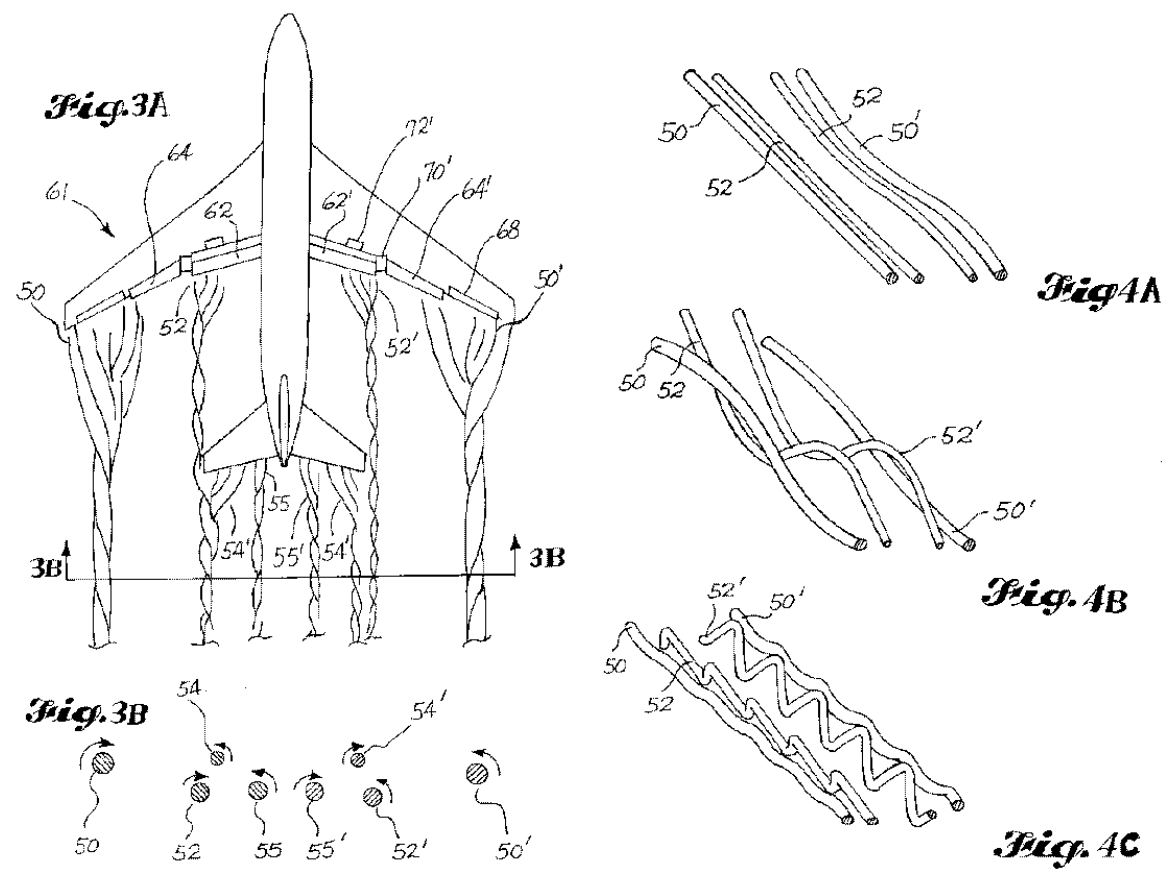

Figure 1: Sketch of vortex system shed by an airplane (left picture) and of long-wave, transient growth and short wave instabilities (right picture, top to bottom) (Courtesy of J. Crouch).

iteration of a so-called one-member strategy can be summarized by

$$
X_{t+1}= \begin{cases}X_{t}+\sigma_{t} Z_{t} & \text { if } f\left(X_{t}+\sigma_{t} Z_{t}\right) \leqslant f\left(X_{t}\right) \\ X_{t} & \text { otherwise }\end{cases}
$$

In the above formula, $Z_{t}$ denotes a random Gaussian vector with zero mean and unit standard deviation. The radius $\sigma_{t}$ of the random walk is updated in function of the success rate at the previous iterations in order to optimize the convergence speed. A high success rate meaning that one is far away from the minimum induces an increase in $\sigma_{t}$. In this work we have implemented the so-called $1 / 5$ rule: the variance is increased if the success ratio during the last iterations is greater than $1 / 5$.

The method just described is isotropic in the sense that the random walk is done in all directions with equal probability. Convergence speed-up can be reached if one adapts the random walk in the direction of best success rate. This is the so-called Covariance Matrix Adaptation (CMA) technique (see [7]). Instead of mutating a single parameter (one-member strategy) one may also consider mutations and recombinations among several individuals (multi-member strategies). For the present purpose, the plain one-member ES proved to be sufficient.

\subsection{Results}

Our study focused essentially on the case of two pairs of co-rotating vortices in the configuration studied by Crouch. The parameters on which the Evolution Strategies optimized were:

- the perturbation initial amplitude of the tip $\left(\epsilon_{1}\right)$ and outboard $\left(\epsilon_{2}\right)$ vortices

- the angles of the perturbation planes $\alpha_{1}, \alpha_{2}$

- the wavelength of the perturbations $\lambda$ 


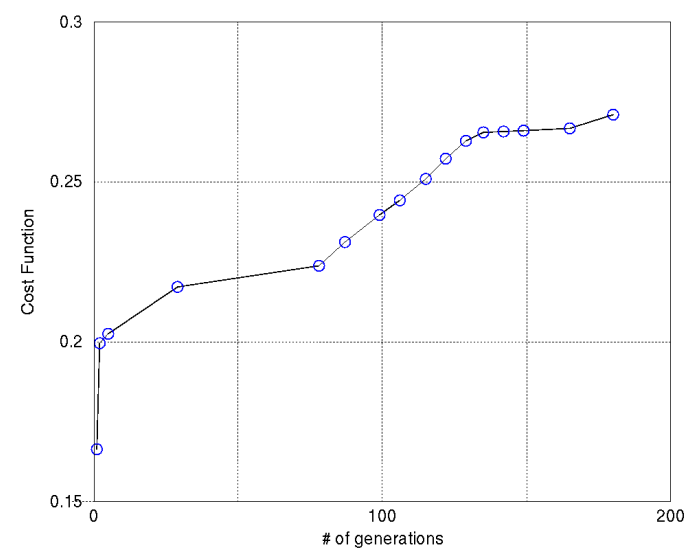

Figure 2: Convergence history for the Evolution Strategy.

- the separation between the two vortices $\delta$

- the circulation ratio between the outboard and tip vortices $\Gamma$

Quantities were adimensionalized by the span (distance between the vorticity centers of each pair) which was always kept constant, and the total circulation of each pair. The computations were done on a periodic box of size $2 \times 2 \times \lambda$ by a vortex method using $64^{3}$ elements (see below in section 3.1 for details on the numerics).

To work with parameters in the same order of magnitude as in [5], the total perturbation was constrained to be below $10 \%$ of the span:

$$
\epsilon_{1}^{2}+\epsilon_{2}^{2} \leqslant 0.01
$$

The following additional constraints were imposed on these parameters to remain within achievable design configurations:

$$
0.25 \leqslant \delta \leqslant 0.4 ; 0.5 \leqslant \lambda \leqslant 10 ; 0 . \leqslant \Gamma \leqslant 0.5
$$

Note that the constraints on $\lambda$ allow for a wide range of wavelengths, varying from short wavelength of the order of a few core sizes, to long wavelengths of the type found in Crow instability.

Our goal was to optimize the instability on the tip, stronger, vortex. To measure the deformation of this vortex, we computed the average angle, inside the core of the tip vortex, of the vorticity vector $\boldsymbol{\omega}$ with the base flow axis. More precisely the objective function was given by the formula

$$
f=\int d z \int_{A(z)} \frac{\omega_{x}^{2}+\omega_{y}^{2}}{\omega_{z}^{2}} d A(z)
$$

where

$$
A(z)=\left\{(x, y),|\boldsymbol{\omega}(x, y, z)| \geqslant 1 / 2|\boldsymbol{\omega}|_{\max }\right\}
$$

Figure 2 shows the convergence history of the Evolution algorithm.

The parameter values finally obtained by the ES are listed on Table 1, together with the parameters that are reported in [5] to lead to efficient transient growth. Some striking similarities can be noticed between these two sets of parameters. In particular the ES has selected perturbations that are mostly located on the tip vortex $\left(\epsilon_{2}<<\epsilon_{1}\right)$, confirming the observation in [5] of efficient transient growth when the outboard vortex was unperturbed. The wavelength of the perturbations $(\lambda)$ is also very close to the one given in [5]. 


\subsection{Perspectives and open questions}

Trailing vortices are a good example of an unsteady complex flow where Evolution Strategies are successful to retrieve optimal parameters. One reason is that the simplicity of the flow geometry enables simulations which are not too expensive. For that particular example, the flexibility of this optimization technique should allow to investigate more complex vortex systems. We actually ran the evolution strategy on 4 pairs, 2 of which being of opposite circulation, playing the role of vortices 54 and 55 in Figure 1. We did obtain parameter values for this system which even improved the optimal growth rate for two pairs. However one open and critical question that remains to be solved is the time span on which the objective should be measured for this particular application. We did observe that, for 4 as well as for 2 pairs, the growth rates significantly decreased after the control time. This confirms observation made in [18] and there is some doubt that these parameters are indeed optimal for fastest reconnection. Moreover the growth rates proved to be rather sensitive to parameter values. The present findings should thus be taken with caution. Nevertheless we believe that the ease Evolution Strategies have to incorporate constraints of any kind in the parameter range, in particular to fit current design constraints, make them an appealing tool for further studies.

\section{Control of three-dimensional wake behind a cylinder}

One important drawback of Evolution strategies for unsteady flows is their computational cost. If, as the above example demonstrated, one-member strategies can be efficient for 3D flows in simple geometries, for more complex problems like drag optimization in bluff-body flows parallel implementations of multi-member strategies are certainly desirable. Moreover, acceptable convergence speed requires to work on parameter spaces as small as possible. For this purpose, it is crucial to accumulate as much knowledge as possible on the flow dynamics in order to select the appropriate parameters to optimize.

We show in the sequel some preliminary results along these lines for the case of a flow past a cylinder. The choice of the cylinder is dictated by the fact that it embodies many generic features of 3D dynamics and that several experimental and numerical results exist to validate the proposed methods. Concerning the particular problem of drag optimization, some results exist in 2D. In particular, optimal control techniques have been applied to propose cylinder rotation or suction and blowing parameters to suppress the shedding [9, 8] or reduce the drag [17]. However to our knowledge these methods have not been extended to 3D flows. Stochastic drag optimization has also been recently used for 2D cylinder [11], and our on-going efforts aim at extending these results to the $3 \mathrm{D}$ case.

Whatever control technique is used, applications to 3D flows are always very demanding on the numerical side. Suction, blowing and body rotations dramatically change the boundary layer dynamics by introducing strong dipoles in the flow. The particle solver that we have used seems to meet the robustness requirements to handle these features. In the sequel we first sketch the numerical code and then discuss some 3D control strategies that give some interesting directions for future optimization.

\begin{tabular}{|l|c|c|c|c|c|c|c|}
\cline { 2 - 8 } \multicolumn{1}{c|}{} & $\alpha_{1}$ & $\alpha_{2}$ & $\epsilon_{1}$ & $\epsilon_{2}$ & $\delta$ & $\Gamma$ & $\lambda$ \\
\hline Optimal parameters & 0.47 & 0.73 & 0.098 & 0.008 & 0.26 & 0.31 & 0.72 \\
\hline Parameters in [5] & $\pi / 4$ & $\pi / 4$ & 0.1 & 0. & 0.3 & 0.4 & 0.7 \\
\hline
\end{tabular}

Table 1: Comparison of the parameters found by the Evolution Strategy and those studied in [5] 


\subsection{A Particle-In-Cell method for 3D bluff-body flows}

We consider the velocity-vorticity form of the incompressible Navier-Stokes equations :

$$
\frac{\partial \boldsymbol{\omega}}{\partial t}+(\mathbf{u} \cdot \boldsymbol{\nabla}) \boldsymbol{\omega}-(\boldsymbol{\omega} \cdot \boldsymbol{\nabla}) \mathbf{u}-\nu \Delta \boldsymbol{\omega}=0
$$

Particle-In-Cell methods combine particles and grids to discretize these equations : particles carry vorticity and implicitly take in account transport terms in the equation, while velocity and strain are computed on an underlying grid. The no-slip boundary condition is enforced through vorticity flux formulas.

Details of these algorithms can be found in $[3,12,2,14,13,4]$. References $[14,15,4]$ more specifically deal with design, validation and results in cylindrical geometry. For a sake of completeness we outline here the main features of the algorithm. Each particle, with index $p$, carries an element of vorticity $\boldsymbol{\omega}_{p}$, with volume $v_{p}$ and location $\mathbf{x}_{p}$. These quantities satisfy the system of differential equations :

$$
\frac{d \mathbf{x}_{p}}{d t}=\mathbf{u}\left(\mathbf{x}_{p}\right), \frac{d \boldsymbol{\omega}_{p}}{d t}=(\boldsymbol{\omega} \cdot \nabla \mathbf{u})\left(\mathbf{x}_{p}\right)+\nu \Delta \boldsymbol{\omega}\left(x_{p}\right)
$$

while volumes remain constant due to the incompressibility. These convection-diffusion equations are solved in a viscous splitting algorithm, alternating convection and diffusion steps.

In the convection step, the particle velocities and strain needed in the right hand side of (2) are obtained as follows: the vorticity carried by the particles is first interpolated on a fixed cylindrical grid and velocity is evaluated by means of grid-based Poisson solvers. More precisely, the velocity is decomposed using the Helmholtz decomposition

$$
\mathbf{u}=\overline{\mathbf{u}}+\nabla \times \psi+\nabla \phi
$$

where $\overline{\mathbf{u}}$ is the irrotational field with the prescribed far field behavior.

The stream function $\psi$ satisfies in the fluid domain $\Omega$ the system

$$
-\Delta \psi=\omega, \nabla \cdot \psi=0
$$

and the potential $\phi$ is used to enforce the no-through boundary condition $\mathbf{u} \cdot \mathbf{n}=0$ - where $\mathbf{n}$ denotes the outward unit normal vector - on the cylinder surface $\Gamma$ :

$$
\Delta \phi=0 \text { in } \Omega, \frac{\partial \phi}{\partial n}=-(\nabla \times \psi) \cdot \mathbf{n} \text { on } \Gamma .
$$

Once velocity ant its derivatives are computed on the grid, these quantities are interpolated back to particles. This allows next to push particles and update their circulations. A fourth order Runge-Kutta time-stepping is used to solve the underlying ordinary differential equations.

After each convection step, particles are remeshed on a regular cylindrical grid. The kernel used to interpolate vorticity from the particles to the grid and to remesh particles is a third order piecewise cubic spline.

Particle vorticities are finally redistributed to simulate diffusion. The no-slip boundary condition $\mathbf{u} \cdot \boldsymbol{\tau}=0$ (imposing a given slip can be done with straightforward modification) is satisfied in the diffusion step through the flux on $\Gamma$ of the tangential components of the vorticity: if $u_{\theta}, u_{z}$ are the tangential components of the velocity in the azimuthal and spanwise directions at the end of an advection step, one solves for the corresponding components of the vorticity the following boundary conditions on the surface of the cylinder

$$
\begin{aligned}
\nu \frac{\partial \omega_{z}}{\partial \mathbf{n}} & =-\frac{u_{\theta}}{\Delta t} \\
\nu\left(\frac{\omega_{\theta}}{r}+\frac{\partial \omega_{\theta}}{\partial \mathbf{n}}\right) & =\frac{u_{z}}{\Delta t}
\end{aligned}
$$



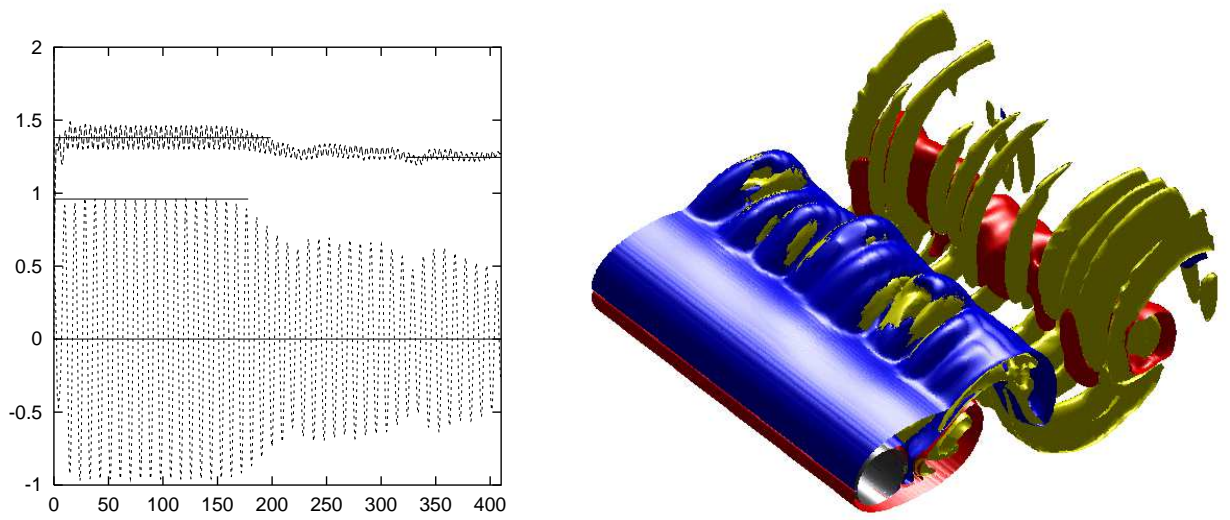

Figure 3: Effect of three-dimensionality on drag and lift coefficients at $R e=300$ (left picture) and vorticity isovalues of post-transient three-dimensional flow at $t=270$ (right picture) (from [15]).

where $\nu$ is the viscosity and $\Delta t$ is the time-step. These vorticity boundary conditions are implemented by means of integral equations.

To summarize, each time-step consists of the following sequence of operations:

- interpolate particle vorticity on a cylindrical grid

- compute stream functions and potential on the grid

- differentiate these quantities on the grid to obtain velocity and strain on the grid

- interpolate velocity and strain back to particles

- push particles and update vorticity

- remesh particles on regular locations

- compute the residual slip on the boundary and diffuse vorticity among particles with the appropriate vorticity fluxes to enforce the desired slip

Several features of wake dynamics can be used to check the accuracy of the method. It is well-known that when the viscosity is small enough (i.e. $R e>190$ ), two-dimensional solutions are unstable and solutions become fully 3D. Figure 3 illustrates the evolution of drag and lift and the production of streamwise vorticity that goes together with the transition from 2D to 3D of the wake for a Reynolds number of 300. For moderate Reynolds numbers the instabilities involved in this transitions are mainly of two kinds, usually called mode $A$ and mode $B$ (see [22]). References [15, 16] show that the present numerical method agrees well in all the classical diagnostics (drag and lift coefficients, Strouhal numbers, exponential growth of instabilities, energy spectrum, spectral profiles of unstable modes, coherent structures) obtained by other numerical or experimental techniques. A nice feature of the method is its combination of robustness and accuracy. Let us point out that robustness was crucial to allow direct numerical simulations even in presence of severe boundary manipulations, as those considered in the sequel.

\subsection{Control strategy using rotation}

As a first example of control for the cylinder wake, we consider the case of spanwise invariant oscillations of the cylinder. It has been shown experimentally in [21] that a fast oscillating rotation of the cylinder leads to 

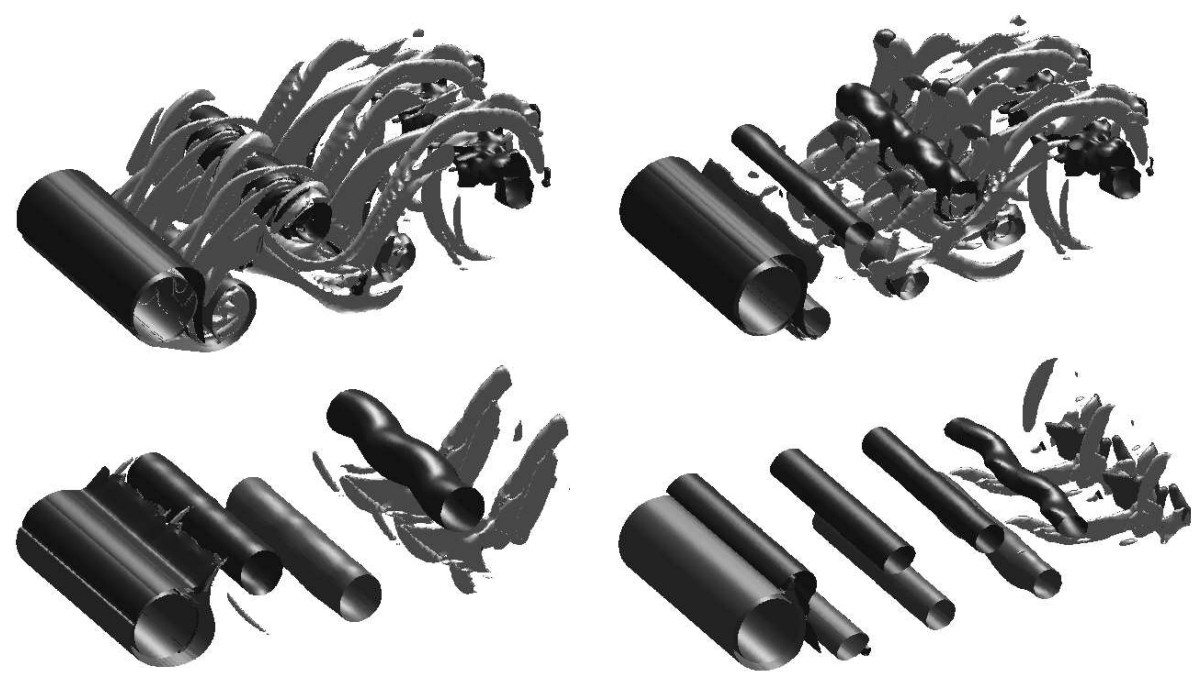

Figure 4: Surfaces of isovorticity after activation of the rotation locked on the flow self-frequency (left pictures) and on twice this frequency (right pictures) (from [15]).

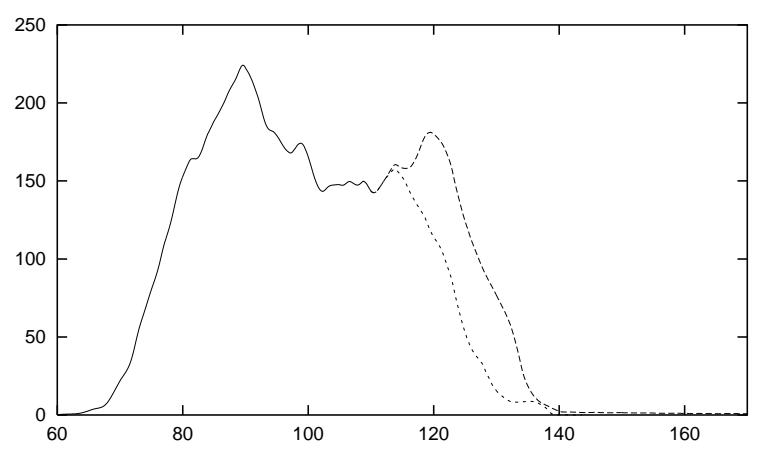

Figure 5: Orthogonal enstrophy versus time for an oscillating rotation of the cylinder. $--:$ rotation lock on the flow self-frequency, - - - : twice the flow self-frequency (from [15]).

a substantial drag reduction. This fact, already observed in 2D simulations [6] has recently been confirmed in fully $3 \mathrm{D}$ simulations $[15,16]$.

An additional property of this kind of control demonstrated in these references is the two-dimensionalization of the flow in a large neighborhood of the body when the angular velocity is large enough. The dimension of the flow is measured by means of directional enstrophies : the spanwise and orthogonal enstrophies

$$
Z_{z}=\int_{\Omega} \omega_{z}^{2} d v, Z^{\perp}=\int_{\Omega} \omega_{x}^{2}+\omega_{y}^{2} d v
$$

represent the amount of vorticity parallel or orthogonal to the cylinder axis. Global enstrophy is recovered by $Z=Z_{z}+Z^{\perp}$.

When $Z^{\perp}$ is small, the flow is mainly two-dimensional, while when $Z^{\perp}$ and $Z_{z}$ are close, the flow is completely three-dimensional. The values of $Z^{\perp}$ obtained at $R e=500$ for an oscillating rotation (half a revolution of amplitude, once and twice the natural frequency of flow) are plotted on figure 5. Isosurfaces are plotted on figure 4 for a control rotation locked on once and twice the flow self-frequency.

In these calculations and in the following, we used a resolution of $256 \times 128 \times 128$ points in the computa- 


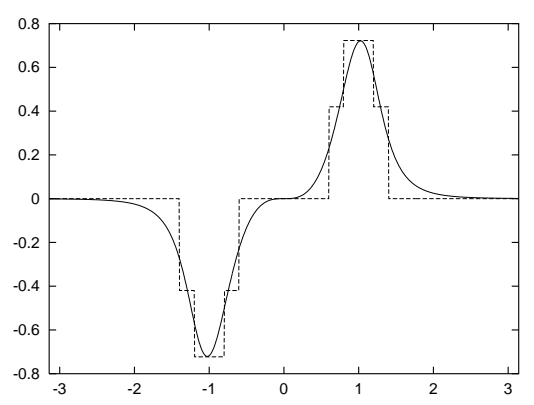

Figure 6: Shape of functions $f$ and $\tilde{f}$, extrema at \pm 0.723 and mid-value at \pm 0.42 .

tional domain

$$
(r, \theta, z) \in \Omega=[1,1+4 \pi] \times[-\pi, \pi] \times[0,4 \pi]
$$

for a cylinder of radius unity. Periodicity was assumed in the spanwise direction.

\subsection{Control strategy using tangential velocity profiles}

In order to propose more effective control strategies, we now consider the case when cylinder rotation is a function of $\theta$ and $z$. Recent results for 2D cylinders [11] have actually allowed to find optimal tangential velocity profiles distributed on 16 points evenly distributed around the cylinder. We chose, as a base profile, a function $f$ that approximately fits these values and allowed spanwise variation along modes $\mathrm{A}$ and $\mathrm{B}$ which describe the 3D instabilities in the wake. Our function $f$ has the following expression

$$
f(\theta)=-\sin \left(\frac{3.2 \theta^{3}}{3+\theta^{10}}\right)
$$

This function, plotted on figure 6, exhibits two extrema, which are chosen near the separation points in order to reduce the drag : the profile $f$ is a smooth function approaching values obtained in [11]. This smooth function can be seen as a regularization of the piecewise constant function $\tilde{f}$ plotted on figure 6 , which could correspond experimentally to a 6-ribbons control.

To account for spanwise variations, the present computations use the four control parameters

$$
\mathbf{C}=\left(\begin{array}{l}
C_{1} \\
C_{2} \\
C_{3} \\
C_{4}
\end{array}\right)
$$

and the azimuthal tangential velocity profile on the body is given by

$$
V_{\text {slip }}(\theta, z)=\frac{D}{2} f(\theta) \mathbf{C} \cdot\left(\begin{array}{c}
1 \\
2 \sin (2 z / D) \\
2 \sin (4 z / D) \\
2 \sin (8 z / D)
\end{array}\right)
$$

with $-\pi \leqslant \theta \leqslant \pi$ and $0 \leqslant z \leqslant 2 \pi D$. The control is started impulsively, at $t_{c}=270$ for $3 \mathrm{D}$ computations.

The energy involved in this control is

$$
E=\frac{1}{2} \int_{0}^{2 \pi D} \int_{-\pi}^{\pi} V_{\text {slip }}(\theta, z)^{2} R d \theta d z=2 \pi R^{3}\|\mathbf{C}\|_{2}^{2} \int_{-\pi}^{\pi} f(\theta)^{2} d \theta
$$

because cross terms have null mean value. The energy required in a particular control within this class is thus given by the Euclidean norm of $C$. 


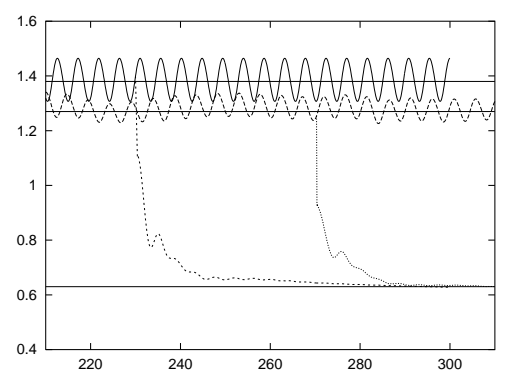

Drag coefficient

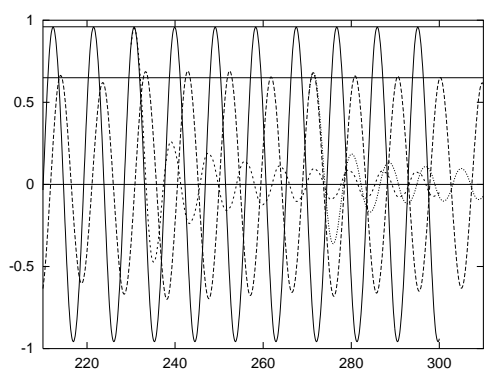

Lift coefficient

Figure 7: Effect of 2D control $\left(C_{1}=1\right)$ on drag and lift coefficients :

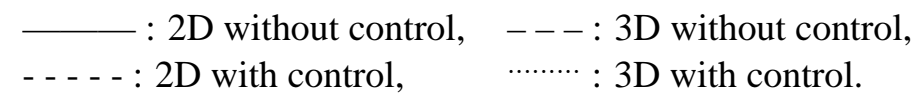

\subsection{Two-dimensional control on $2 \mathrm{D}$ and $3 \mathrm{D}$ wakes}

By two-dimensional control, we mean a velocity profile that does not depend on spanwise coordinate, and thus can be applied to both 2D and 3D flows.

This implies that the control parameter set is $\mathbf{C}=\left(C_{1}, 0,0,0\right)$. Figure 7 shows that the mean drag coefficient, at $R e=300$, drops from 1.38 down to 0.64 - a $53 \%$ reduction - when $C_{1}=1$. The effect of such a control on the vorticity field is plotted on figure 8 , at different time from $t_{c}=270$ to $t_{c}+50=320$. One may observe that, as for the case of constant rotation, the flow returns to a 2D state, and that a substantial stretching of the recirculation zone occurs.

\subsection{Control with 3D vorticity profiles}

In order to first analyze individually the effects on the wake of various 3D forcings, we consider the following control vectors:

- $\mathbf{C}=(0,1,0,0)$, with wavelength $\lambda / D=\pi$, matching the mode $A$ instability wavelength at $R e=300$ (called type A control in the sequel),

- $\mathbf{C}=(0,0,1,0)$, with wavelength $\lambda / D=\pi / 2$ intermediate between mode $A$ and mode $B$ wavelength (type A-B control),

- $\mathbf{C}=(0,0,0,1)$, with wavelength $\lambda / D=\pi / 4$, matching the mode $B$ instability wavelength (type $B$ control).

- $\mathbf{C}=(1,0,0,0)$, spanwise invariant (2D-type control).

All these controls involve the same energy. The case $\mathbf{C}=(1,0,0,0)$ has already been discussed. As an example, type A-B control points are shown on figure 9.

Figure 10 contains snapshots of the vorticity field for 2 different cases. It shows to which extent the forcing affects the topology of the wake.

Finally, combination of modes are considered as control functions. We focus our attention on combination of 2D-type and A-B type controls, involving the same energy as in the last section. 


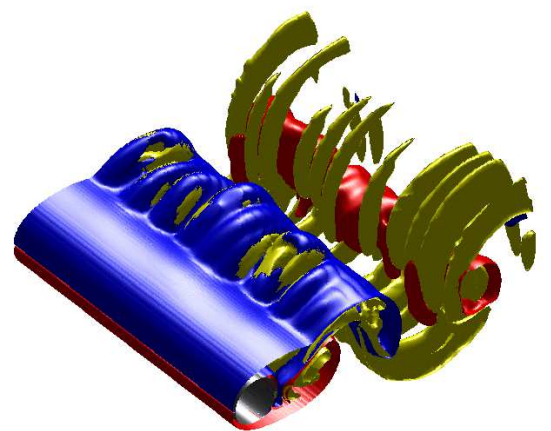

$t=270$

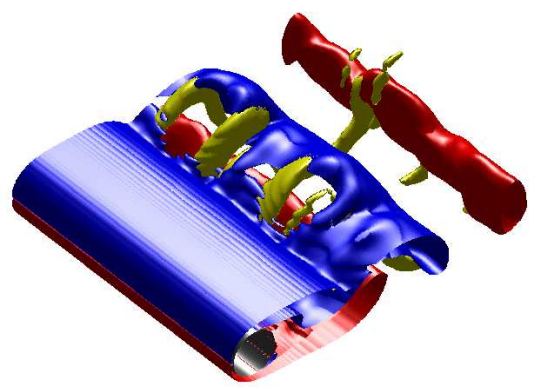

$t=290$

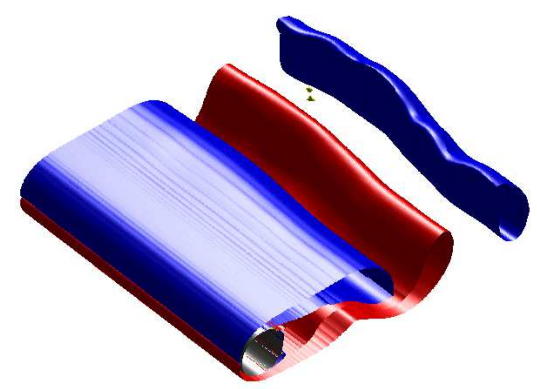

$t=310$

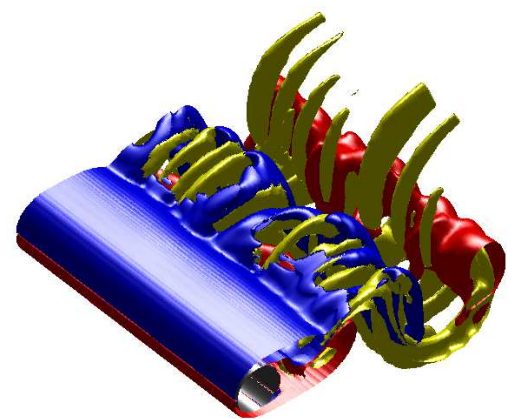

$t=280$

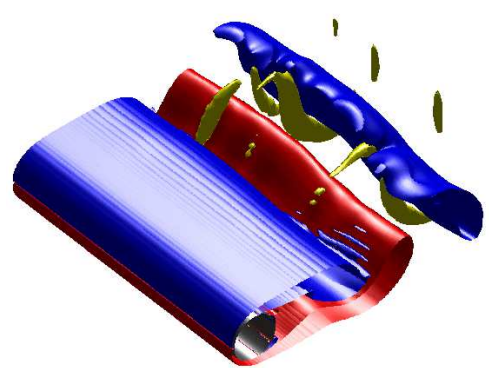

$t=300$

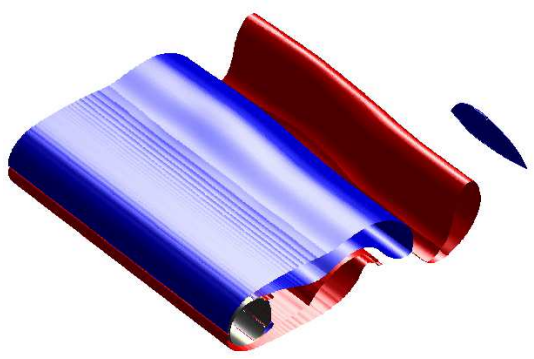

$t=320$

Figure 8: Effect of the two-dimensional control $C_{1}=1$ : vorticity field for a sequence of times.
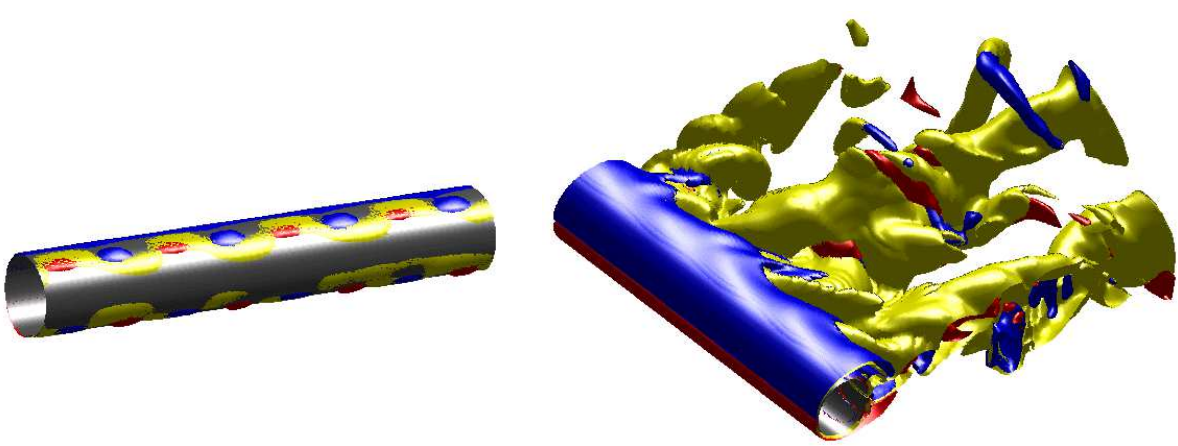

Figure 9: Type A-B control : control points (left picture) and resulting flow at $t=380=t_{c}+110$ (right picture). 


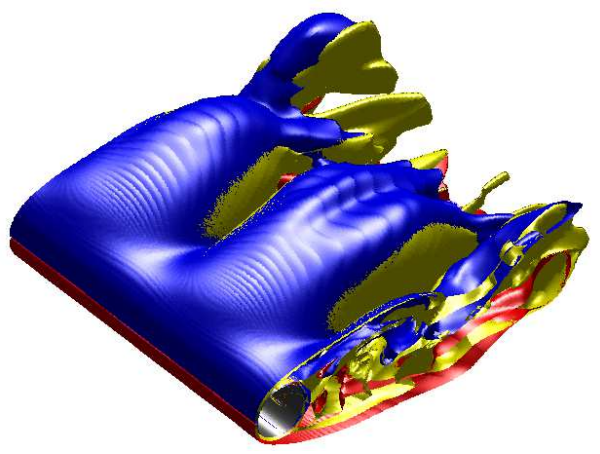

$\lambda / D=\pi, t=320$

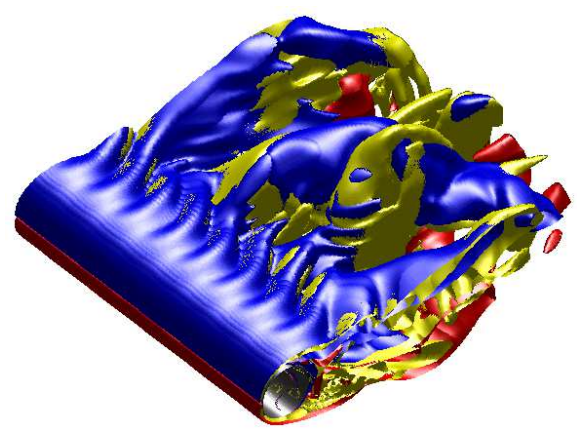

$\lambda / D=\pi / 4, t=320$

Figure 10: Influence of control wavelength on the vorticity field.

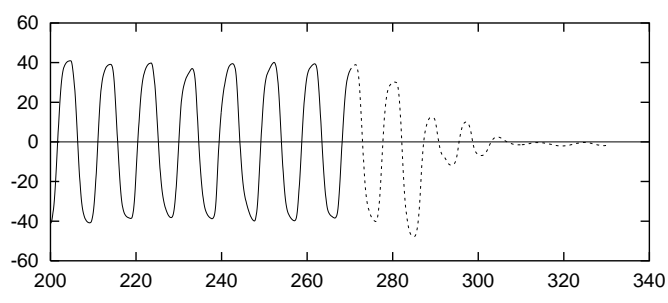

Figure 11: Mean spanwise vorticity $\Gamma_{z}$ versus time, using mixed control (type A-B and 2D) on a 3D flow. : without control, - - - - : control on 3D flow.

This leads to the parameters

$$
\mathbf{C}=\frac{1}{\sqrt{5}}(2,1,0,0)
$$

which means that the velocity has the following expression :

$$
V_{\text {slip }}(\theta, z)=\frac{D}{\sqrt{5}}(1+\sin (2 z / D)) f(\theta)
$$

This profile, whose wavelength is $\lambda / D=\pi / 2$, has a very special property. It breaks the von Kármán streets, into streamwise structures of vorticity which find a quasi-stationary state, thus preventing the shedding of the flow. To measure the amount of shedding, one can consider the time evolution of the mean spanwise vorticity

$$
\Gamma_{z}=\int_{\Omega} \omega_{z} d v
$$

When von Kármán alleys leave the computational domain (i.e. in presence of shedding), this quantity oscillates. A lack of oscillation, as seen in Figure 11, thus shows an absence of shedding. Figure 13 shows the vorticity isosurface corresponding to this particular type of wake.

Drag curves corresponding to an impulsively started control on either a 2D or a fully developed 3D wake, are plotted on figure 12. A comparison of this result with Figure 7 suggests that the suppression of shedding, resulting from a 3D forcing, induces an additional drag reduction of about $10 \%$ over a purely $2 \mathrm{D}$ control. This preliminary result brings some confidence that a fully $3 \mathrm{D}$ optimization might improve $2 \mathrm{D}$ results. 


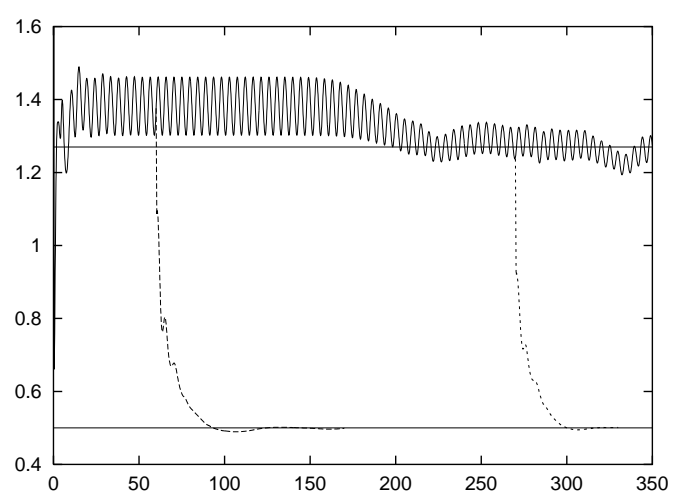

Figure 12: Drag coefficient versus time, using mixed control (type A-B and 2D) on 2D and 3D flows. without control, - - - : control on 2D flow, - - - : control on 3D flow. Constant values at 0.5, 1.27 and 1.38.

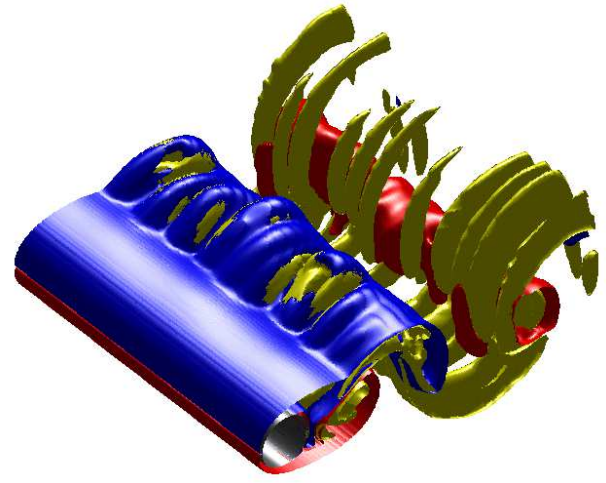

$t=t_{c}=270$

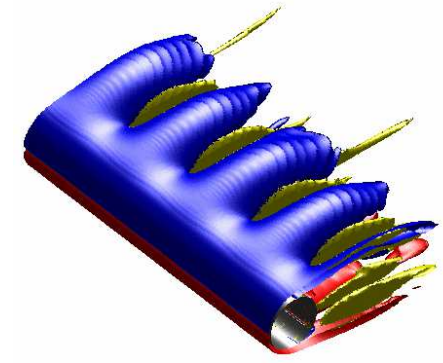

$t=t_{c}+30$

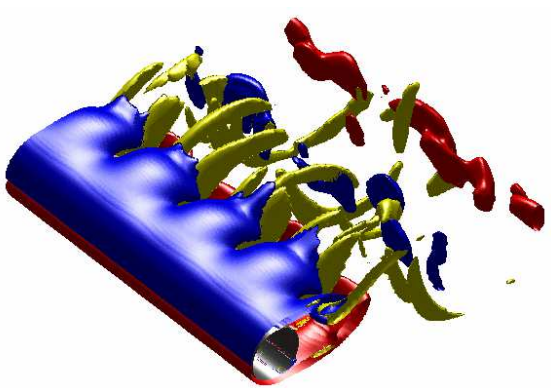

$t=t_{c}+10$

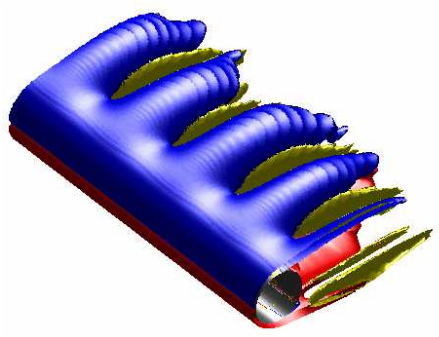

$t=t_{c}+60$

Figure 13: Isosurface of vorticity, using mixed control applied on 3D flow. 


\section{Conclusion and outlook}

We have considered two examples of wake optimization: the destruction of trailing vortices and the reduction of drag in a cylinder wake. The first example was simple enough, from the computational point of view, to allow reasonably fast optimization by stochastic optimization. Furthermore, this class of optimization techniques proved to be particularly flexible and opens the possibility to incorporate more closely design constraints. In the second case, the complexity of the flow dynamics makes a brute force stochastic optimization far too expensive. A reduction of the number of parameters is crucial. Open loop control emphasizing the role of type A and type B instabilities give promising results which should open the way to efficient implementations of fully 3D optimization algorithms.

\section{Acknowledgments}

The authors gratefully acknowledge many stimulating discussions with M. Milano and P. Koumoutsakos that have made this work possible. The first part of of the work also benefited from discussions with K. Shariff and P. Spalart. The Evolution Strategy code used in the first part was developed by S. Mueller at ETH-Zürich, and the computational ressources for the second part of the work were provided by the joint UJF-CEA project CIMENT. The first author was partially supported by the Center for Turbulence research at Stanford University and NASA Ames.

\section{References}

[1] Cottet, G.-H., Sbalzarini, I., Mueller, S.D. \& Koumoutsakos, P. Optimization of trailing vortices destruction by evolution strategies. Center for Turbulence Research, Proceedings of the 2000 Summer Program.

[2] Cottet, G.-H., Michaux, B., Ossia, S. \& Vanderlinden, G. A comparison of spectral and vortex methods in three-dimensional incompressible flows J. Comp. Phys. 2002; 175:1-11.

[3] Cотtet G.-H., Koumoutsakos P. Vortex methods, theory and practice. Cambridge University Press 2000.

[4] Cottet, G.-H. \& Poncet, P. Advances in Particle-In-Cell methods for the simulation of 3D bluff-body flows. Submitted 2002.

[5] Crouch, J.D. Instability and transient growth for two trailing-vortex pairs. J. Fluid Mech. 1997 350:311330.

[6] Dennis, S.C.R., Nguyen, P. \& Kocabiyik, S. The flow induced by a rotationally oscillating and translating circular cylinder. J. Fluid Mech. 2000; 385:255-286.

[7] Hansen, N. \& Ostermeier, A. Adapting arbitrary normal mutation distribution in evolution strategies: the covariance matrix adaptation. Proceedings of the 1996 IEEE Int. Conf. on Evolutionary Computation:312-317.

[8] Homescu, C., NAvon, I.M. \& Li, Z. Suppression of vortex shedding for flow around a circular cylinder using optimal control. Int. J. Numer. Fluids. 2002; 38(1):43-69.

[9] Li, Z., Navon, I.M., Hussaini, M.Y. \& Le Dimet, F.-X. Optimal Control of Cylinder Wakes via Suction and Blowing. Computers and Fluids 2002; in press. 
[10] Koumoutsakos P. Vorticity flux control in a turbulent channel flow. Phys. Fluids 1999; 11(2):248-250.

[11] Milano M., Koumoutsakos P. A clustering genetic algorithm for cylinder drag optimization. J. Comput. Phys. 2002; 175(1):79-107.

[12] Ould-Sahili M.L., Cottet G.-H. \& El Hamraoui M. Blending finite-differences and vortex methods for incompressible flow computations. SIAM J. Sci. Comput. 2000; 22:1655-1674.

[13] Ploumhans P,, Wincklemans G., Salmon J., Leonard A., Warren M. Vortex methods for high-resolution simulation of three-dimensional bluff-body flows; applications to the sphere at $\mathrm{Re}=300$, 500 and 1000 J. Comp. Phys. 2002; 178:427-463.

[14] Poncet P. Méthodes particulaires pour la simulation des sillages tridimensionnels. PhD thesis, University Joseph Fourier, Grenoble, France, 2001.

[15] Poncet P. Vanishing of mode B in the wake behind a rotationnaly oscillating circular cylinder. Phys. of Fluids. 2002; 14(6):2021-2024.

[16] PONCET P. Topological aspects of the three-dimensional wake behind rotary oscillating circular cylinder. Under revision for J. Fluid Mech. 2003.

[17] Protas B.\& Styczek, A. Optimal rotary control of the cylinder wake in the laminar regime. Phys. of Fluids. 2002; 14(7):2073-2087.

[18] RENNICH, S.C. \& LELE S.K. A method for accelerating the destruction of aircraft wake vortices AIAA Paper 1998;98-0667.

[19] Sbalzarini, I, Mueller, S., Koumoutsakos, P. \& Cottet, G.-H. Evolution Strategies for Computational and Experimental Fluid Dynamics Applications, Proceedings of the Genetic and Evolutionary Computation Conference (GECCO-2001), Morgan Kaufmann Publishers, San Francisco, 2001.

[20] SPAlart, P.R. Airplane trailing vortices Ann. Rev. Fluid Mech. 1998; 30:107-138.

[21] Tokumaru P \& Dimotakis P. Rotary oscillation control of a cylinder wake. J. Fluid Mech. 1991; 224:77-90.

[22] Williamson C.H.K. Three-dimensional wake behind a cylinder. J. Fluid Mech. 1996; 328:345. 